\title{
Demonstration of potential link between Helicobacter pylori related promoter CpG island methylation and telomere shortening in human gastric mucosa
}

\author{
Tomomitsu Tahara ${ }^{1}$, Tomoyuki Shibata ${ }^{1}$, Masaaki Okubo ${ }^{1}$, Tomohiko Kawamura ${ }^{1}$, \\ Noriyuki Horiguchi ${ }^{1}$, Takamitsu Ishizuka ${ }^{1}$, Naoko Nakano', Mitsuo Nagasaka ${ }^{1}$, \\ Yoshihito Nakagawa ${ }^{1}$, Naoki Ohmiya ${ }^{1}$ \\ ${ }^{1}$ Department of Gastroenterology, Fujita Health University School of Medicine, Toyoake, Japan \\ Correspondence to: Tomomitsu Tahara, email: tomomiccyu@yahoo.co.jp \\ Keywords: DNA methylation, telomere length, gastric mucosa, H. pylori, gastritis \\ Received: October 24, $2015 \quad$ Accepted: May 02, $2016 \quad$ Published: June 01, 2016
}

\section{ABSTRACT}

Background: Telomere length shortening in Helicobacter pylori (H. pylori) infected gastric mucosa constitutes the earliest steps toward neoplastic transformation. In addition to this genotoxic changes, epigenetic changes such as promoter CpG island (PCGI) methylation are frequently occurred in $\boldsymbol{H}$. pylori infected gastric mucosa. The aim of this study was to investigate a potential link between $\boldsymbol{H}$. pylori related PCGI methylation and telomere length shortening in the human gastric mucosa.

Methods: Telomere length was measured in non-neoplastic gastric mucosa from 106 cancer-free subjects. To identify $\boldsymbol{H}$. pylori related PCGI methylation, bisulfite pyrosequencing was used to quantify the methylation of 49 PCGIs from 47 genes and LINE1 repetitive element

Results: We identified five PCGIs (IGF2, SLC16A12, SOX11, P2RX7 and MYOD1), which the methylation is closely associated with $H$. pylori infection. Hypermethylation of all these PCGIs was associated with development of pathological state from normal to mild, active, and atrophic gastritis $(P<0.001)$ and lower pepsinogen $I /$ II ratio $(P<0.05)$, an indicator for gastric mucosal atrophy. Telomere shortening was significantly associated with mean $Z$ score methylation of five PCGIs $(R=-0.39$, $P<0.0001$ ) and four of these locus (IGF2: $R=-0.35, P=0.0003, S L C 16 A 12: R=-0.35$, $P=0.0002, P 2 R X 7: R=-0.29, P=0.003$, and MYOD1: $R=-0.33, P=0.0005$ ). Multivariate analysis revealed that telomere shortening held an increased risk for hypermethylation (odds ratio: 1.71, 95\% confidence interval: 1.11-2.63, $P=0.016$ ).

Conclusion: Potential link between $\boldsymbol{H}$. pylori related PCGI methylation and telomere shortening emphasize the importance of genotoxic-epigenetic interaction in the pathological state of $\boldsymbol{H}$. pylori infected gastric mucosa.

\section{INTRODUCTION}

Telomeres consist of repetitive nucleotide sequences and an associated terminal protein complex that help avoid loss of chromosomal integrity [1]. Telomere shortening in genomic DNA seems to reflect lifetime accumulative oxidative stress through environmental exposures [2-6]. Shortened telomere lengths were also observed in human epithelial cancers due to the formation of complex nonreciprocal translocations and increased chromosome instability [7-10]. Telomere length abnormalities also occur early in epithelial carcinogenesis, suggesting that telomere length may serve as a useful biomarker in human cancers.

Helicobacter pylori $(H$. pylori) infected gastric mucosa is characterized as chronic inflammation [11, 12], which is closely linked with telomere length shortening $[13,14]$. These facts suggest that shortened telomere constitutes the earliest steps toward neoplastic transformation relevant to gastric cancer predisposition. In addition to this genotoxic changes, epigenetic alterations may also play a role in this process. For example, regional promoter $\mathrm{CpG}$ island (PCGI) hypermethylation and global DNA hypo methylation are frequently occurred in 
H. pylori infected gastric mucosa in relation to severity of inflammation and/or atrophy $[15,16]$. Our preliminary result showed that telomere shortening correlated with DNA methylation at $C D H 1$ gene promoter [17]. Induction of methylation in the gastric mucosa by $H$. pylori involves complex biological processes that are still not completely understood. The aim of this study was to investigate a potential link between $H$. pylori related PCGI hypermethylation and telomere length shortening in the human gastric mucosa.

\section{RESULTS}

\section{Identification of hyper methylated PCGIs in $H$. pylori positive gastric mucosa and their relationship with severity of $\boldsymbol{H}$. pylori related gastritis}

Age and sex distributions, $H$. pylori infection status, prevalence of peptic ulcer disease and status of histological gastritis are shown in Table 1 . To explore hyper methylated PCGIs in $H$. pylori positive gastric mucosa, we initially performed unsupervised hierarchical clustering analysis of 49 PCGIs and LINE1 among six H. pylori negative and six positive cases (Figure 1). This analysis showed that all $H$. pylori positive gastric mucosa was clearly clustered as hyper methylated samples compared with the $H$. pylori negative mucosa. Based on this result, we selected five candidate PCGIs (IGF2, SLC16A12, SOX11, P2RX7 and MYOD1) and LINE1 repetitive element for further analysis. As expected, hypermethylation of all five PCGIs were tightly linked to $H$. pylori infection status (all $P<0.0001$, Figure 2). In line with previous result showing correlation between DNA methylation and $H$. pylori related gastritis [16], development of pathological state from normal to mild, active, and atrophic gastritis was correlated with hypermethylation of all these PCGIs (all $P<0.001$, Figure $\mathrm{S} 1$ ). We also showed that lower PG I/II ratio that indicates the severity of gastric mucosal atrophy was significantly associated with hypermethylation of all these PCGIs (all $P<0.05$, Figure S2).

We also observed that Methylation of three PCGIs (SLC16A12, SOX11 and MYOD1) were also associated with increasing age (all $P<0.05$, Figure S3), while this association seemed smaller compared to the associations between methylation status and $H$. pylori infection, degree of histological gastritis and PG I/II ratio.

\section{Telomere shortening in $\mathrm{H}$. pylori related gastritis and its relationship with PCGI methylation}

Telomere length was significantly correlated with H. pylori infection and histological gastritis (Figure S4), which is in line with our resent study [17]. We found that telomere shortening was significantly associated with mean $\mathrm{Z}$ score methylation of selected five PCGIs $(\mathrm{R}=$ $0.39, P<0.0001$, Figure 3). In detail, methylation of four out of all five locus significantly associated with telomere shortening (IGF2: $\mathrm{R}=-0.35, P=0.0003, S L C 16 A 12$ : $\mathrm{R}=-$ $0.35, P=0.0002, P 2 R X 7: \mathrm{R}=-0.29, P=0.003$, and $M Y O D 1$ : $\mathrm{R}=-0.33, P=0.0005$, Figure 4). LINE1 repetitive element, an indicator for genome wide hypo methylation has been shown to be associated with $H$. pylori related enlarged fold gastritis and PCGI methylation [25]. We also found significant association between LINE1 hypo methylation and mean $\mathrm{Z}$ score methylation of all these PCGIs $(\mathrm{R}=$ $0.21, P=0.03$, Figure 3 ) as well as three out of all five locus (IGF2: $\mathrm{R}=-0.26, P=0.008, S O X 11: \mathrm{R}=-0.19, P=0.05$, and MYOD1: $\mathrm{R}=-0.21, P=0.03$, Figure S5) but this association seemed to be marginal when compared to the association with telomere shortening (Figure 3 and 4). To assess the factors related to DNA methylation in the gastric mucosa, univariate and multivariate analysis were performed. Since the mean $\mathrm{Z}$ score methylation of all five PCGIs in the gastric mucosa presented an approximately Gaussian distribution, with over representation of methylation-high cases (data not shown), we set cut-off values of 0.3 (mean $\mathrm{Z}$ score methylation) for the definition of methylation-high cases. Univariate analysis revealed that age (odds ratio: 1.04, 95\% confidence interval: 1.00-1.07, $P=0.04$ ), $H$. pylori positive (odds ratio: $34.3,95 \%$ confidence interval: 4.44-264.62, $P=0.0007$ ), duodenal ulcer (odds ratio: 3.70, 95\% confidence interval: $1.04-13.20, P=0.04$ ), atrophic gastritis (odds ratio: $4.67,95 \%$ confidence interval: $2.02-$ $10.80, P=0.0003$ ) and telomere length shortening (odds ratio: $1.96,95 \%$ confidence interval: $1.35-2.84, P=0.0004$ ) were significantly associated with methylation-high. (Table 2) Multivariate analysis of these factors revealed that age (odds ratio: $1.05,95 \%$ confidence interval: 1.01 1.09, $P=0.03$ ), $H$. pylori positive (odds ratio: $15.26,95 \%$ confidence interval: $1.78-130.97, P=0.013$ ), duodenal ulcer (odds ratio: $7.68,95 \%$ confidence interval: $1.43-$ 41.20, $P=0.0174$ ) were significantly associated with methylation-high. We observed that telomere length shortening was also remained as an independent factor associated with methylation-high (odds ratio: 1.71, 95\% confidence interval: $1.11-2.63, P=0.016$ ). (Table 3 )

\section{DISCUSSION}

Although the mechanisms of methylation induction in the gastric mucosa by H. pylori are not completely understood, chronic inflammation, pro-inflammatory cytokines and oxidative stress may have a role in this process [26-28]. We have selected five PCGIs (IGF2, SLC16A12, SOX11, P2RX7 and MYOD1) from cancer and inflammation/age related panels. Methylation of these genes well correlated with $H$. pylori infection, histological and serological gastritis, which is consistent with previous studies $[15,16]$. We showed that these PCGI hypermethylation well correlated with telomere 
Table 1: Clinic-pathological features of subjects

\begin{tabular}{lc}
\hline Variables & \\
\hline Total number of subjects: $\mathrm{n}$ & 106 \\
Mean age: +/-SD & $59.4+/-13.1$ \\
Gender: male n (\%) & $64(60.4 \%)$ \\
H. pylori positives: $\mathrm{n}(\%)$ & $75(70.8 \%)$ \\
Gastric ulcer: $\mathrm{n}(\%)$ & $15(14.2 \%)$ \\
Duodenal ulcer: $\mathrm{n}(\%)$ & $12(11.3 \%)$ \\
Normal gastric mucosa: n (\%) & $15(14.2 \%)$ \\
Mild gastritis: $\mathrm{n}(\%)$ & $21(19.8 \%)$ \\
Active gastritis: $\mathrm{n}(\%)$ & $24(22.6 \%)$ \\
Atrophic gastritis: $\mathrm{n}(\%)$ & $46(43.4 \%)$ \\
\hline
\end{tabular}

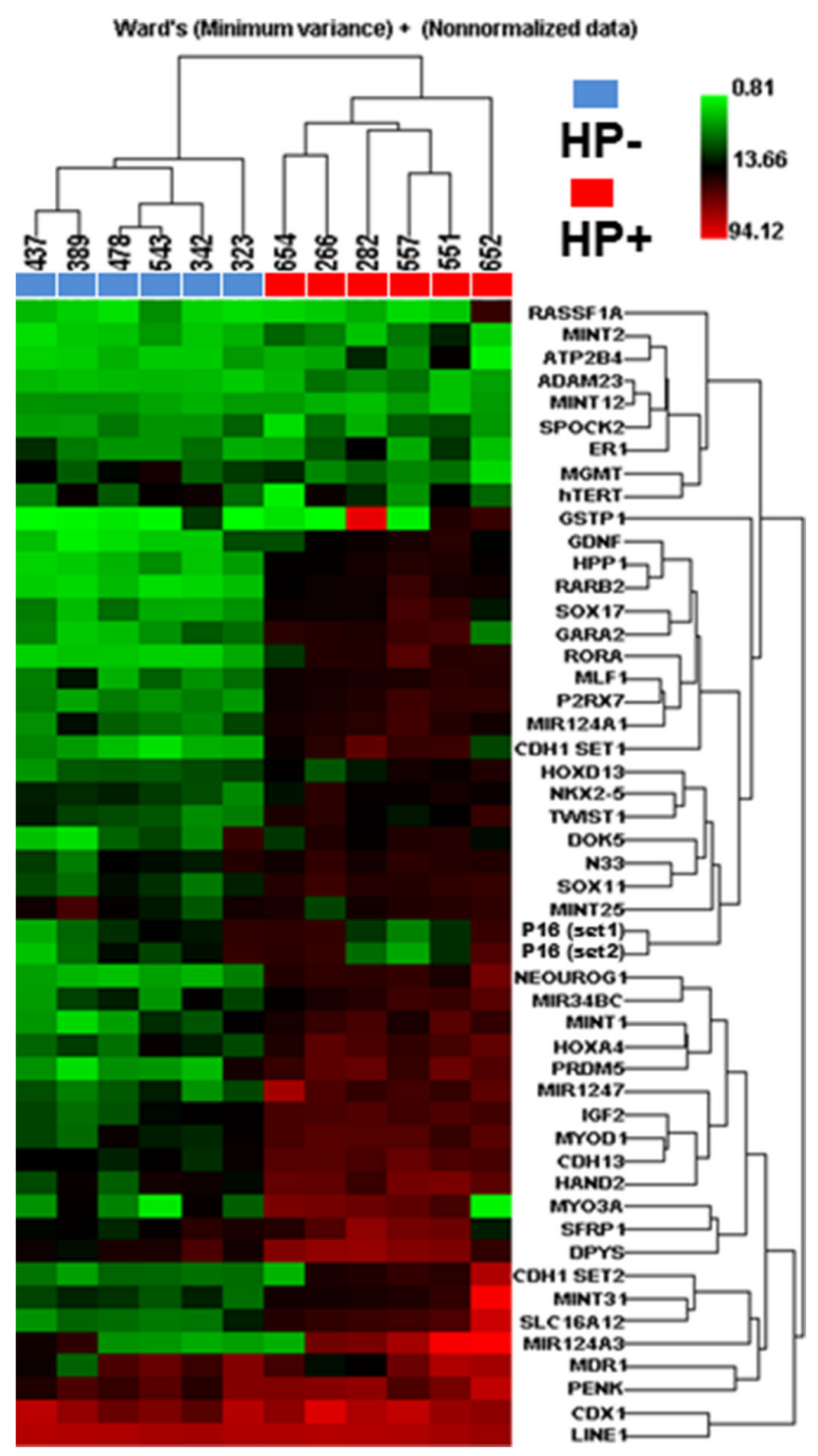

Figure 1: Unsupervised hierarchical clustering analysis of 49 PCGIs and LINE1 repetitive element among six $H$. pylori negative (blue boxes) and six positive cases (red boxes). Numbers listed above the blue and red boxes indicates sample ID. 

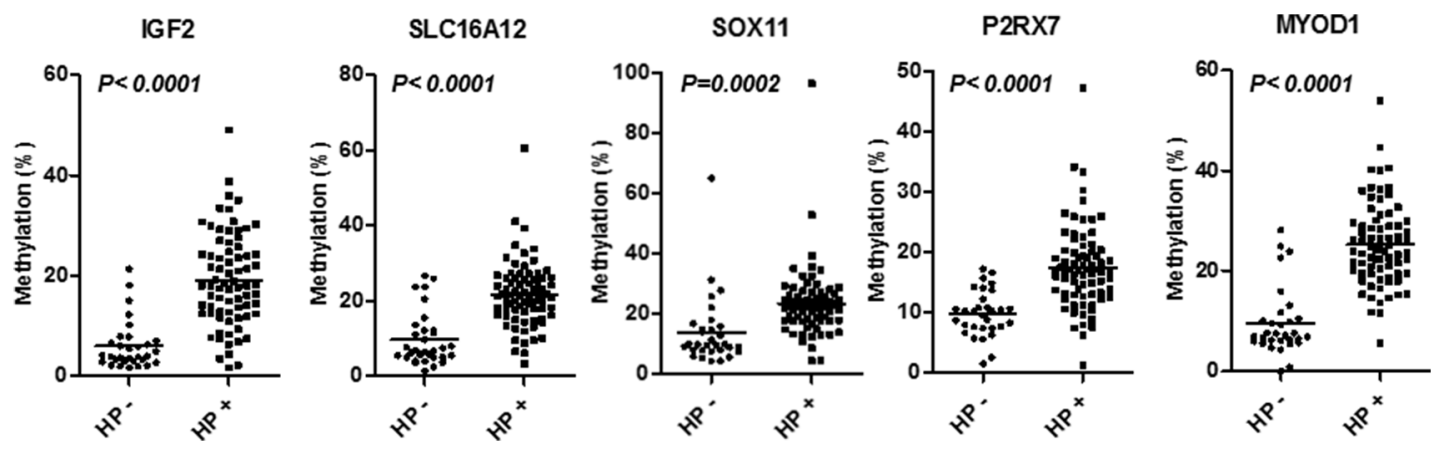

Figure 2: Methylation status of five PCGIs (IGF2, SLC16A12, SOX11, P2RX7 and MYOD1) among all subjects with or without $\boldsymbol{H}$. pylori infection. Horizontal bars represents mean methylation percentage. The statistical analysis was performed using Student's t-Test.
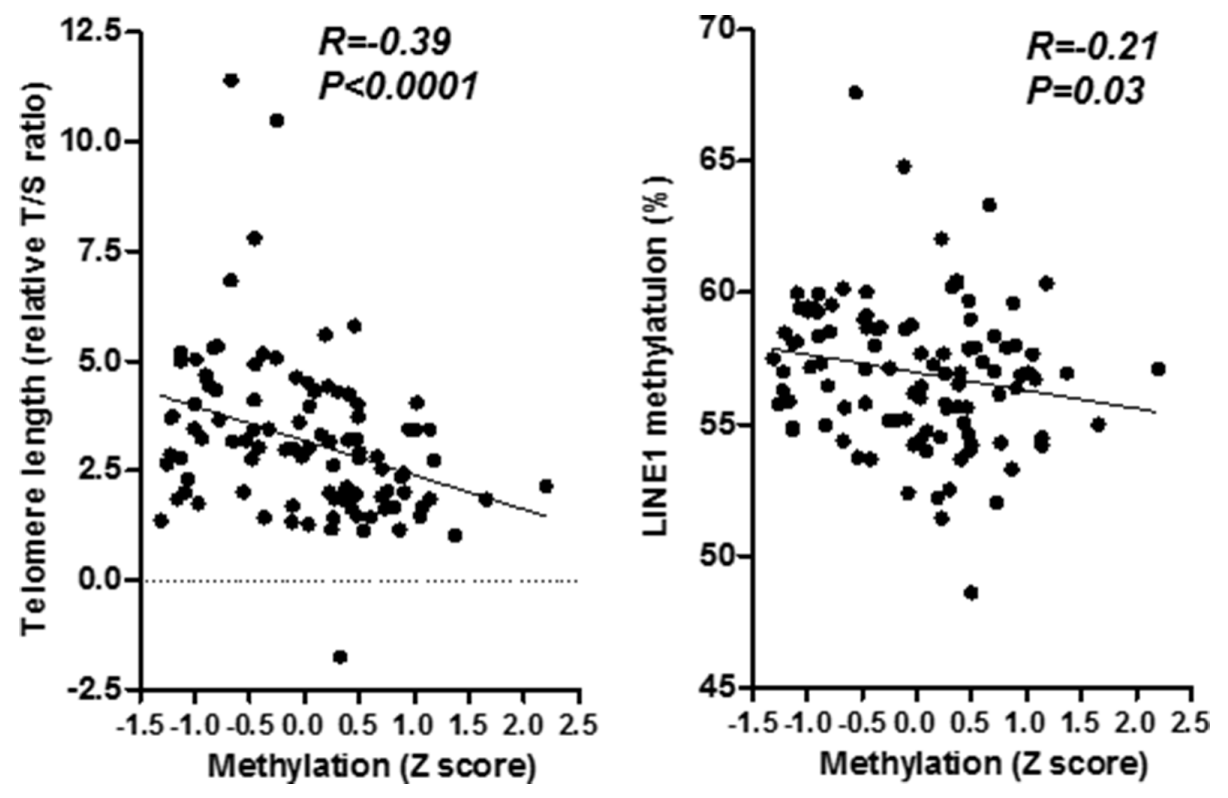

Figure 3: Association between telomere shortening and mean $\mathrm{Z}$ score methylation of five PCGIs (left) and methylation of $L I N E 1$ repetitive element (right). Statistical analysis was performed using the Spearman correlation analysis.
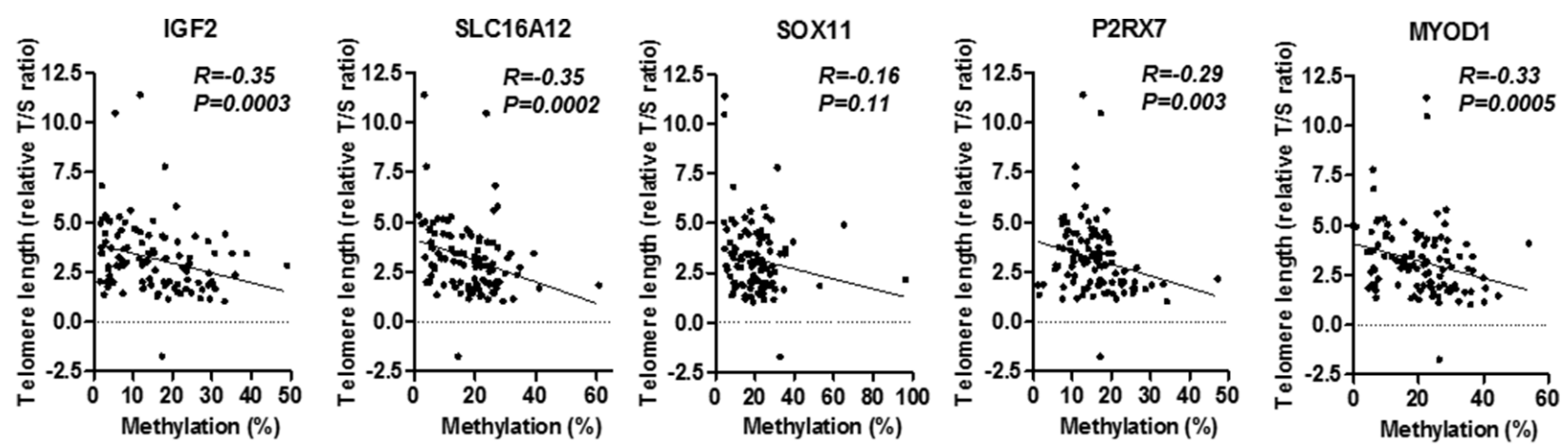

Figure 4: Association between telomere shortening and methylation status of five PCGIs (IGF2, SLC16A12, SOX11, $P 2 R X 7$ and $M Y O D 1)$ Statistical analysis was performed using the Spearman correlation analysis. 
Table 2: Univariate analysis assessing the factors related to the methylation high

\begin{tabular}{lcc}
\hline Variables & Odds ratio (95\% confidence interval) & P value \\
\hline Age & $1.04(1.00-1.07)$ & 0.04 \\
Gender (male) & $0.88(0.40-1.96)$ & 0.76 \\
H. pylori positive & $34.3(4.44-264.62)$ & 0.0007 \\
Gastric ulcer & $2.01(0.67-6.04)$ & 0.21 \\
Duodenal ulcer & $3.70(1.04-13.20)$ & 0.04 \\
Active gastritis & $1.47(0.58-3.68)$ & 0.41 \\
Atrophic gastritis & $4.67(2.02-10.80)$ & 0.0003 \\
Telomere shortening & $1.96(1.35-2.84)$ & 0.0004 \\
LINE1 methylation & $1.09(0.94-1.27)$ & 0.24 \\
\hline
\end{tabular}

*Methylation high, mean $\mathrm{Z}$ score of methylation $>0.3$.

Table 3: Multivariate analysis assessing the factors related to the methylation high

\begin{tabular}{lcc}
\hline Variables & Odds ratio (95\% confidence interval) & P value \\
\hline Age & $1.05(1.01-1.09)$ & 0.03 \\
H. pylori positive & $15.26(1.78-130.97)$ & 0.013 \\
Duodenal ulcer & $7.68(1.43-41.20)$ & 0.017 \\
Atrophic gastritis: n (\%) & $2.08(0.70-6.20)$ & 0.19 \\
Telomere shortening & $1.71(1.11-2.63)$ & 0.016 \\
\hline
\end{tabular}

*Methylation high, mean $\mathrm{Z}$ score of methylation $>0.3$.

shortening. Hypermethylation of all individual locus as well as their mean $\mathrm{Z}$ score was closely associated with telomere shortening. Multivariate analysis demonstrated that telomere length shortening is an independent factor associated with PCGI hypermethylation in the gastric mucosa. Our result emphasize the importance of genotoxic-epigenetic interaction in the pathological state of $H$. pylori infected gastric mucosa. Telomere shortening was identified in inflammatory tissues such as $H$. pyloripositive gastric epithelial tissue and colonocytes in ulcerative colitis $[13,14,17,29]$, while it is also observed in aged peripheral leukocyte DNA. Thus, it seems telomeres shortening is a consequence of cell replication and oxidative damage [30]. There have been report showing potential link between telomere shortening and DNA methylation in other cell types. Telomere shortening is correlated with promoter methylation profile of $\mathrm{p} 16 /$ $\mathrm{Rb}$ and $\mathrm{p} 53 / \mathrm{p} 21$ pathways in breast cancer tissue [31]. In blood cells, biological age predicted by DNA methylation status rather than chronological age is well correlated more with telomere length shortening and acquired aplastic anemia or dyskeratosis congenita - two diseases associated with progressive bone marrow failure [32]. The link between telomere shortening and subtelomeric
DNA methylation was also confirmed in vitro using human fibroblasts cultured under conditions of chronic oxidative stress, induced by tert-butyl hydroperoxide [33]. Together with link between $H$. pylori related PCGI methylation and telomere shortening in the stomach, it is suggested that alteration of telomere shortening and DNA methylation predict various pathological state and clinical outcomes across the different tissue types. Since H. pylori infected gastric mucosa enhances chronic inflammation and increased cell turnover, it is reasonable to expect the relationship between PCGI hypermethylation and telomere shortening. On the other hand, direct mechanistic link of PCGI hypermethylation and telomere shortening has not been clearly demonstrated so far [34, 35]. Further investigation will be needed to clarify this issue.

LINE1 repetitive element, an indicator for genome wide hypo methylation has been shown to be associated with $H$. pylori related enlarged fold gastritis, which is a form of gastritis with an increased risk of gastric cancer [25]. The LINE1 hypo methylation is also associated with PCGI hypermethylation in certain genes, such as $C D H 1, C D H 13$, and PGP9.5 genes [25]. We also found significant association between LINE1 hypo methylation and $\mathrm{CpG}$ island promoter methylation but this association 
seemed to be marginal compared to the association with telomere shortening. One merit in our study is we carefully selected PCGI methylation closely linked to $H$. pylori infection. The result suggest that $H$. pylori related $\mathrm{CpG}$ island promoter methylation is correlated more with telomere shortening than genome wide hypo methylation. Since telomere shortening and increased PCGI hypermethylation in $H$. pylori infected gastric mucosa is likely to be an outcome of chronic inflammation or increased cell turnover, $H$. pylori related gastritis can be therefore viewed as premature aging of gastric epithelial cells. Chronic inflammation in H. pylori infected gastric mucosa leads to telomere shortening and increased PCGI hypermethylation, which may eventually lead to cancer progression. The potential usefulness of telomere shortening and related $\mathrm{CpG}$ island hypermethylation as the molecular marker for gastric cancer needs to be evaluated in various clinical settings. Recent comprehensive genome wide methylation analysis identified PCGIs at which methylation levels are associated with leukocyte telomere length. The result demonstrated that the associated sites were enriched in subtelomeric loci (within $4 \mathrm{Mb}$ of the telomere), and also at loci in imprinted regions [36]. Further investigations to elucidate the relationship between telomere length and DNA methylation should be needed to understand the biological importance of genotoxic-epigenetic interaction in pathological state of human gastric mucosa.

\section{MATERIALS AND METHODS}

\section{Subjects}

Enrolled were 106 subjects who were attending the Endoscopy Center of Fujita Health University Hospital between January 2005 and April 2010. All subjects performed upper gastroscopy for various indications such as health check, secondary complete check-up of stomach cancer following to barium X-ray examination, followup examination of ulcer diseases, or for the complaint of abdominal discomfort. Patients who had severe systemic disease, malignancy in the stomach or other organ, previous history of $H$. pylori eradication as well as continuous usage of non-steroidal anti-inflammatory drugs were not included. The cohort was partly recruited from previous studies investigating the genetic polymorphisms, and telomere length among gastroduodenal diseases [17-19]. The Ethics Committee of the Fujita Health University School of Medicine approved the protocol, and prior, written informed consent was obtained from all participating subjects.

\section{DNA extraction, and detection of $\boldsymbol{H}$. pylori infection, histological assessment}

During upper gastroscopy, biopsy specimens were taken from the antrum and the upper corpus along the greater curvature from grossly non-pathological mucosa in all patients. The specimens taken from the antrum were cut into two pieces, and one part of specimens was immediately frozen and stored at $-80^{\circ} \mathrm{C}$ until for the molecular experiment. Genomic DNA was extracted directly from these frozen specimens using the standard protein precipitating method. Other specimens were fixed in $10 \%$ buffered formalin and embedded in paraffin for microscopic histological examination. Using hematoxylin and eosin-stained histological slides, the extent of chronic inflammation, atrophy, and metaplasia in the antrum were assessed according to the updated Sydney system [20], with each factor being scored from 0 to 3 ( 0 , normal; 1 , mild; 2 , moderate; 3 , severe). This assessment was performed by senior pathologists in our hospital who were blinded to the characteristics of subjects. Based on this scoring system, subjects were divided in to following four categories; Normal, all 0 ; Active gastritis, 2 or higher score of acute or chronic inflammation; Atrophic gastritis, 2 or higher score of acute or chronic inflammation; Mild gastritis, all others. Histological assessment also confirmed that all the specimens contained more than $70 \%$ of epithelial cells. Assessment of $H$. pylori infection status was performed using two biopsy specimens taken from the antrum and the upper corpus by immunohistochemistry using the polyclonal rabbit anti-Helicobacter pylori antibody (FLEX Anti-Helicobacter Pylori, Code GA523, Dako, Tokyo, Japan).

\section{Serological evaluation}

The serum pepsinogen (PG) I/II ratio was calculated based on the data of the serum PG I and PG II levels measured by radioimmunoassay in 80 cases. PG I/II ratio that showed a decrease in proportion to the severity of gastric mucosal atrophy [21 22] was used as a marker of atrophic gastritis.

\section{Relative average telomere length measurement}

Relative telomere length was measured as a comparative quantification, in particular as abundance of telomeric template vs. a single-copy gene $(\mathrm{T} / \mathrm{S})$ by quantitative real-time PCR as described previously. [23] For the quantitative real-time PCR, the iTaq SYBR Green Supermix (Bio-Rad) and ABI Prism 7900HT RealTime PCR System (Applied Biosystems) were used. The primers for telomeres and single-copy genes (h-globin) are listed in Supplementary Table 2. Detailed protocol of experiment is available in our recent study [17].

\section{Selection of candidate panels and PCGI methylation analysis by bisulfite pyrosequencing}

Bisulfite pyrosequencing was used to quantify the methylation of 49 PCGIs from 47 genes. We reasoned 
that there might be two approaches to selecting genes (all selected from the literature), one based on frequency of methylation in gastric and other cancers (MINT1, MINT2, MINT12, MINT25, MINT31, RORA, GDNF, PRDM5, MLF1, CDH1, p16 etc.) and a separate one based on methylation in non-neoplastic inflamed/ aged tissues (ER, CDH13, MYOD1, SFRP1, P2RX7, IGF2, N33, MIR124A1, MIR124A3 etc.) [24]. We also evaluated the methylation status of the LINE1 repetitive element using bisulfite pyrosequencing. Bisulfite-treated genomic DNA was used to evaluate the methylation status by the bisulfite pyrosequencing. Bisulfite treatment of DNA was carried out with BislFast DNA Modification Kit (Toyobo, Osaka, Japan) according to the manufacturer's protocol. Pyrosequencing was carried out using PSQ24 system with Pyro-Gold reagent Kit (QIAGEN, Tokyo, Japan), and the results were analyzed using PyroMark Q24 software (QIAGEN). The primers used for pyrosequencing are listed in Supplementary Table S1.

\section{Data analysis}

Using 12 gastric mucosa derived from six $H$. pylori negative and six positive patients, an unsupervised hierarchical clustering analysis was used to identify distinct subgroups based on the methylation status of 49 PCGIs and LINE1 repetitive element. Continuous variables between two groups was assessed using the Student's t-Test. Continuous variables among more than two groups were assessed using the One way ANOVA. The correlation of continuous variables between two groups was assessed using the Spearman correlation analysis. Univariate and multivariate analysis were also performed to assess the factors related to PCGI methylation. P value $<0.05$ was considered statistically significant.

\section{CONFLICTS OF INTEREST}

We declare that we have no conflicts of interest.

\section{GRANT SUPPORT}

This work was supported by JSPS KAKENHI Grant-in-Aid for Young Scientists (B) Grant Number JP26870685.

\section{REFERENCES}

1. de Lange T. The protein complex that shapes and safeguards human telomeres. Genes Dev 2005;19:2100-10.

2. Jennings BJ, Ozanne SE, Hales CN. Nutrition, oxidative damage, telomere shortening, and cellular senescence: individual or connected agents of aging? Mol Genet Metab 2000;71:32-42.

3. von Zglinicki T. Oxidative stress shortens telomeres. Trends Biochem Sci ;27:339-44.
4. Valdes AM, Andrew T, Gardner JP, Kimura M, Oelsner E, Cherkas LF, Aviv A, Spector TD. Obesity, cigarette smoking, and telomere length in women. Lancet 2005;366:662-4.

5. Wu X, Amos CI, Zhu Y, Zhao H, Grossman BH, Shay JW, Luo S, Hong WK, Spitz MR. Telomere dysfunction: a potential cancer predisposition factor. J Natl Cancer Inst 2003;95:1211-8.

6. Schönland SO, Lopez C, Widmann T, Zimmer J, Bryl E, Goronzy JJ, Weyand CM. Premature telomeric loss in rheumatoid arthritis is genetically determined and involves both myeloid and lymphoid cell lineages. Proc Natl Acad Sci U S A 2003;100:13471-6.

7. Artandi SE, Chang S, Lee SL, Alson S, Gottlieb GJ, Chin L, DePinho RA. Telomere dysfunction promotes nonreciprocal translocations and epithelial cancers in mice. Nature 2000;406:641-5.

8. Bailey SM, Murnane JP. Telomeres, chromosome instability and cancer. Nucleic Acids Res 2006;34:2408-17.1

9. Cheung AL, Deng W. Telomere dysfunction, genome instability and cancer. Front Biosci 2008;13:2075-90.

10. Murnane JP. Telomeres and chromosome instability. DNA Repair (Amst) 2006;5:1082-92.

11. Parsonnet J. Helicobacter pylori and gastric cancer. Gastroenterol Clin North Am 1993;22:89-104.

12. Peek RM, Jr., Blaser MJ. Helicobacter pylori and gastrointestinal tract adenocarcinomas. Nat Rev Cancer 2002;2:28-37.

13. Aida J, Izumiyama-Shimomura N, Nakamura K, Ishii A, Ishikawa N, Honma N, Kurabayashi R, Kammori M, Poon SS, Arai T, Takubo K. Telomere length variations in 6 mucosal cell types of gastric tissue observed using a novel quantitative fluorescence in situ hybridization method. Hum Pathol 2007;38:1192-200.

14. Moran AP, Knirel YA, Senchenkova SN, Widmalm G, Hynes SO, Jansson PE. Helicobacter pylori infection is closely associated with telomere reduction in gastric mucosa. Oncology 2003;65:275-82.

15. Maekita T, Nakazawa K, Mihara M, Nakajima T, Yanaoka K, Iguchi M, Arii K, Kaneda A, Tsukamoto T, Tatematsu M, Tamura G, Saito D, Sugimura T, et al. High levels of aberrant DNA methylation in Helicobacter pylori-infected gastric mucosae and its possible association with gastric cancer risk. Clin Cancer Res. 2006;12:989-95.

16. Tahara T, Arisawa T, Shibata T, Nakamura M, Yoshioka D, Okubo M, Maruyama N, Kamano T, Kamiya Y, Fujita H, Nakagawa Y, Nagasaka M, Iwata M, et al. Increased number of methylated $\mathrm{CpG}$ islands correlates with Helicobacter pylori infection, histological and serological severity of chronic gastritis. Eur J Gastroenterol Hepatol. 2009;21:613-9.

17. Tahara T, Shibata T, Kawamura T, Ishizuka T, Okubo M, Nagasaka M, Nakagawa Y, Arisawa T, Ohmiya N, Hirata I. Telomere length in non-neoplastic gastric mucosa and its relationship to $\mathrm{H}$. pylori infection, degree of gastritis, and NSAID use. Clin Exp Med. 2015 [Epub ahead of print] 
18. Nomura $T$, Tahara $T$, Shiroeda $H$, Minato $T$, Matsue Y, Hayashi R, Matsunaga K, Otsuka T, Nakamura M, Toshikuni N, Shibata T, Arisawa T. Influence of HRH2 promoter polymorphism on aberrant DNA methylation of DAPK and CDH1 in the gastric epithelium. BMC Gastroenterol. 2013;13:1.

19. Yamada H, Tahara T, Shiroeda H, Hayashi R, Saito T, Nakamura M, Tsuchishima M, Tsutsumi M, Shibata T, Arisawa T. Effects of $-1018 \mathrm{G}>\mathrm{A}$ polymorphism of $\mathrm{HRH} 2$ (rs2607474) on the severity of gastric mucosal atrophy. J Gastrointestin Liver Dis. 2012;21:139-43.

20. Dixon MF, Genta RM, Yardley JH, Correa P. Classification and grading of gastritis: the updated Sydney system. International Workshop on the Histopathology of Gastritis, Houston 1994. AmJ Surg Pathol 1996;20:1161-81.

21. Kekki M, Samloff IM, Varis K, Ihamäki T. Serum pepsinogen I and serum gastrin in the screening of severe atrophic corpus gastritis. Scand J Gastroenterol 1991;186:109-16.

22. Asaka M, Kimura $\mathrm{T}$, Kudo M, Takeda H, Mitani S, Miyazaki T, Miki K, Graham DY. Relationship of Helicobacter pylori to serum pepsinogens in an asymptomatic Japanese population. Gastroenterology 1992;102:760-6.

23. Cawthon RM. Telomere measurement by quantitative PCR. Nucleic Acids Res 2002;30:e47

24. Tahara T, Arisawa T. DNA methylation as a molecular biomarker in gastric cancer. Epigenomics. 2015;7:475-86.

25. Yamamoto E, Toyota M, Suzuki H, Kondo Y, Sanomura T, Murayama Y, Ohe-Toyota M, Maruyama R, Nojima M, Ashida M, Fujii K, Sasaki Y, Hayashi N, et al. LINE-1 hypomethylation is associated with increased $\mathrm{CpG}$ island methylation in Helicobacter pylori-related enlargedfold gastritis. Cancer Epidemiol Biomarkers Prev. 2008; 17:2555-64.

26. Wang M, Furuta T, Takashima M, Futami H, Shirai N, Hanai H, Kaneko E. Relation between interleukin-1beta messenger RNA in gastric fundic mucosa and gastric juice $\mathrm{pH}$ in patients infected with Helicobacter pylori. J Gastroenterol. 1999; 34:10-7.

27. Yamaoka Y, Kita M, Kodama T, Sawai N, Kashima K, Imanishi J. Induction of various cytokines and development of severe mucosal inflammation by cagA gene positive Helicobacter pylori strains. Gut. 1997;41:442-51.
28. Meyer-ter-Vehn T, Covacci A, Kist M, Pahl HL. Helicobacter pylori activates mitogen-activated protein kinase cascades and induces expression of the proto-oncogenes c-fos and c-jun. J Biol Chem. 2000;275:16064-72.

29. Risques RA, Lai LA, Himmetoglu C, Ebaee A, Li L, Feng Z, Bronner MP, Al-Lahham B, Kowdley KV, Lindor KD, Rabinovitch PS, Brentnall TA. Ulcerative colitis-associated colorectal cancer arises in a field of short telomeres, senescence, and inflammation. Cancer Res. 2011;71:1669-79.

30. Cheng Y. Desreumaux P. 5-Aminosalicylic acid is an attractive candidate agent for chemoprevention of colon cancer in patients with inflammatory bowel disease. World J Gastroenterol 2005;11:309-14.

31. Radpour R, Barekati Z, Haghighi MM, Kohler C, Asadollahi R, Torbati PM, Holzgreve W, Zhong XY. Correlation of telomere length shortening with promoter methylation profile of $\mathrm{p} 16 / \mathrm{Rb}$ and $\mathrm{p} 53 / \mathrm{p} 21$ pathways in breast cancer. Mod Pathol. 2010;23:763-72.

32. Weidner CI, Lin Q, Koch CM, Eisele L, Beier F, Ziegler P, Bauerschlag DO, Jöckel KH, Erbel R, Mühleisen TW, Zenke M, Brümmendorf TH, Wagner W. Aging of blood can be tracked by DNA methylation changes at just three CpG sites. Genome Biol. 2014;15:R24.

33. Boesten DM, de Vos-Houben JM, Timmermans L, den Hartog GJ, Bast A, Hageman GJ. Accelerated aging during chronic oxidative stress: a role for PARP-1. Oxid Med Cell Longev. 2013;2013:680414.

34. Choudhury SR, Cui Y, Milton JR, Li J, Irudayaraj J. Selective increase in subtelomeric DNA methylation: an epigenetic biomarker for malignant glioma. Clin Epigenetics. 2015;7:107.

35. Zhang ZX1, Wang Y, Tao ZZ, Chen SM, Xiao BK, Zhou T. Subtelomeric demethylation deregulated hTERT expression, telomerase activity, and telomere length in four nasopharyngeal carcinoma cell lines. Cancer Biother Radiopharm. 2014;29:289-94.

36. Buxton JL, Suderman M, Pappas JJ, Borghol N, McArdle W, Blakemore AI, Hertzman C, Power C, Szyf M, Pembrey M. Human leukocyte telomere length is associated with DNA methylation levels in multiple subtelomeric and imprinted loci. Sci Rep. 2014;4:4954. 